\title{
Variations in apparent spatial frequency with stimulus orientation: I. Incidence of the effect in the general population
}

\author{
D. O. BOWKER \\ Center for Visual Science, University of Rochester, Rochester, New York 14627
}

\begin{abstract}
The human visual system is anisotropic not only for stimulus detection and discrimination but also for stimulus appearance. The apparent length of a stationary stimulus and the apparent velocity of a moving stimulus vary with orientation. These variations are predictable from a model assuming a compression of the horizontal spatial meridian relative to the vertical meridian. In this paper, the apparent spatial frequency of a suprathreshold grating is shown to depend on the grating orientation. Specifically, horizontal gratings appear coarser than vertical gratings of equivalent spatial frequency. Additional data collected from the same group of observers show that horizontal lines appear shorter than vertical lines of the same extent. The presence of the spatial frequency and length illusions, although both predictable from the compression model, were not found to be correlated within observers. Implications of these results for other data in the literature are discussed.
\end{abstract}

The human visual system is anisotropic; that is, it often exhibits different characteristics to stimuli presented in different orientations. The most familiar class of visual anisotropy involves those cases in which human visual function is in some way superior for the detection and recognition of stimuli oriented horizontally or vertically than for stimuli oriented obliquely. A number of studies have demonstrated reduced acuity (Emsley, 1925; Leibowitz, 1953; Ogilvie \& Taylor, 1958) and grating contrast sensitivity (Campbell, Kulikowski, \& Levinson, 1966; Freeman \& Thibos, 1975; Mitchell, Freeman, \& Westheimer, 1967) for stimulus patterns presented in oblique orientations. In addition, discrimination thresholds for both orientation (Matin \& Drivas, 1979; Taylor, 1963) and spatial frequency (Bowker, 1980) are higher for obliquely oriented stimuli than for horizontal or vertical stimuli. Many of these "oblique effects" (Appelle, 1972) are specific to higher spatial frequencies (Bowker, 1980; Campbell, Kulikowski, \& Levinson, 1966), and several studies have shown them to remain after the optics of the eye have been bypassed with laser interference techniques (Freeman \& Thibos, 1975; Mitchell, Freeman, \& Westheimer, 1967).

A second class of visual anisotropy involves differences between the horizontal and vertical meridians. A number of 19 th century authors, notably Fick

Thanks are extended to R. M. Chapman, R. N. Haber, R. M. Springer, and $\mathbf{W}$. Makous for their comments and criticisms of this research project. This research was funded in part by National Eye Institute Grant EY-01319 awarded to the Center for Visual Science. The author's address after June 1981: Department of Ophthalmology, University of Wisconsin, Room 177, Medical Sciences, Madison, Wisconsin 53706.
(1851), Helmholtz (1866), and Wundt (1862), observed that vertical lines tend to look longer than horizontal lines of equivalent extent. This finding has been replicated by several investigators in the more recent past (Finger \& Spelt, 1947; Pearce \& Matin, 1969; Pollack \& Chapanis, 1952; Ritter, 1917; Shipley, Nann, \& Penfield, 1949; Thompson \& Schiffman, 1974). In addition, it has been observed that vertically moving targets tend to have a higher apparent velocity than horizontally moving targets (Brown, 1931; Avery \& Day, 1971). Taken together, these studies indicate that there may be a perceptual compression of the horizontal meridian of space relative to the vertical meridian. If this were true, it would certainly have implications for the suprathreshold appearance of other classes of stimuli in different orientations.

In this paper, results are presented showing that for a majority of human observers, the apparent spatial frequency of a grating depends on its orientation. Specifically, horizontal gratings tend to appear coarser, that is, of a lower spatial frequency, than their vertical counterparts. In addition, data were collected on the presence or absence of the standard horizontalvertical length illusion in these same observers. If the horizontal-vertical length illusion arises from the same underlying influences that produce horizontalvertical differences in apparent spatial frequency, these measures should be correlated within observers.

The orientation-contingent distortions in apparent spatial frequency are shown by Bowker (1981) to occur over a wide range of stimulus spatial frequencies and contrasts, and are shown to remain when the grating patterns are imaged directly on the retina with laser interference techniques. 


\section{METHOD}

\section{Observers}

Fifty-eight observers were obtained from an introductory psychology course taught at the University of Rochester. Subjects received course credit for their participation in the experiment. The sign-up sheet requested that only subjects with no astigmatism participate in the experiment, although 5 subjects did have to be excluded on the basis of astigmatism. When the subjects arrived at the room in which the experiment was conducted, they were initially asked if they wore contacts or glasses or had any other visual problems of which they were aware. Of the 58 volunteers, 22 wore corrective lenses of some kind. The other subjects reportedly had normal vision. If subjects did wear corrective lenses, the experimenter examined them to determine if any had an astigmatic correction. The data from 5 subjects were excluded for this reason, although these data were collected and these observers were given credit for their participation. Twenty-five of the remaining observers were male and 28 were female. Ages ranged from 17 to 22 years $(\bar{X}=18.5$ years, $\sigma=.88$ years $)$.

\section{Procedure}

In the first part of the experiment, observers were shown 30 pairs of square-wave gratings via a Kodak 650 Carousel slide projector. Observers viewed the stimuli binocularly through natural pupils, and their observation distance was held constant at $225 \mathrm{~cm}$ using a chinrest. Within each grating pair, one pattern was always vertical and the other was always horizontal. In 12 of the slides the spatial frequencies of the two gratings were identical (5.49 cycles/deg), in 9 the vertical grating was coarser (12\% spatial frequency difference), and in the other 9 the horizontal grating was coarser. Gratings were presented in laterally placed circular configurations (Figure 1). Circular grating patterns had a diameter of $3.80 \mathrm{deg}$ and a center-to-center separation of $4.75 \mathrm{deg}$. Grating contrast was $91 \%$, and mean luminance was $22.3 \mathrm{~cd} / \mathrm{m}^{2}$.

Observers, upon presentation of each grating pair, had to choose the coarser of the two gratings. Observers were told that even if they could not tell a difference, they should make their best guess. They were also encouraged to give their first impressions as to which grating looked coarser. As soon as the observer made a response, the slide projector was advanced and the next slide was exposed. Subjects generally responded within $5 \mathrm{sec}$ of stimulus exposure.

Following the $\mathbf{3 0}$ gratings exposures, subjects were allowed a brief rest and were given instructions about the second part of the experiment. Following this interlude, observers were shown a series of 24 slides. Each slide contained a vertically oriented line and a horizontally oriented line arranged in a cross-hair configuration (Figure 2). Of the 24 line pairs, 8 were of identical length (8.2 deg visual angle), in 8 the vertical line was longer (12\% length difference), and in 8 the horizontal line was longer. Observers were instructed to choose which of the two lines was longer even if they had to guess.
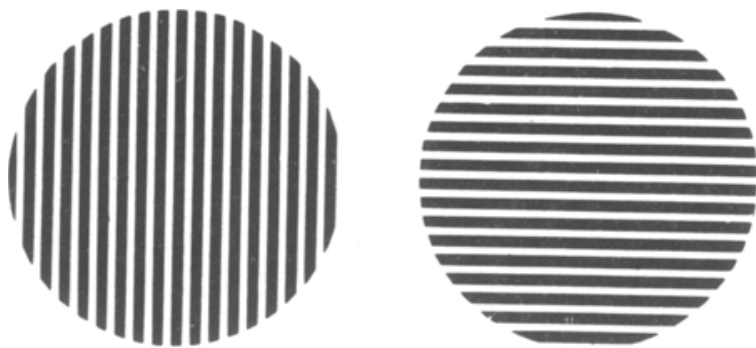

Figure 1. Sample grating stimulus used in forced-choice experiment. Grating diameter equals 3.80 deg. Center-to-center separation equals 4.75 deg.

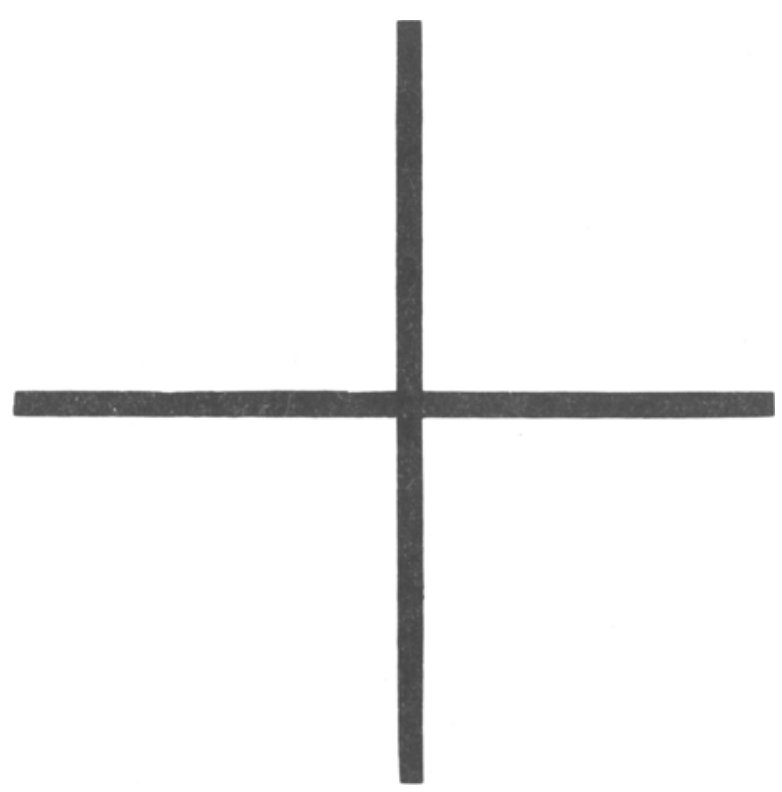

Figure 2. Sample line stimulus used in second part of experiment.

\section{RESULTS}

Figure 3 shows a frequency histogram of the number of observers choosing the horizontal gratings as being coarser a given number of times when the two gratings were actually the same spatial frequency. If observers were just as likely to pick the horizontal gratings as the vertical gratings, the distribution should balance on the dashed line. Clearly, the distribution is heavily weighted to the right of this line, indicating that most observers chose the horizontal grating as coarser most of the time. To further examine the hypothesis that observers were just as likely to choose the horizontal grating as they were the vertical grating in the judgment of pattern coarseness, the following statistical procedure was employed. A binomial distribution with a mean of 6 $\left[\overline{\mathrm{X}}_{\mathrm{bin}}=\mathrm{Np}=12(.5)=6\right]$ and a standard deviation of $1.73\left(o_{\text {bin }}=\sqrt{\mathrm{Npq}}=\sqrt{12(.5)(.5)}=1.73\right)$ was assumed. This distribution provided an expected number of individuals (of 53) for each bin in the frequency histogram shown in Figure 3. This hypothetical distribution was then compared with the actual distribution by a chi-square analysis. The chi-square statistic was computed to be $7709.60(\mathrm{df}=13)$, which has a probability well below the .001 level. The hypothesis that the probability of a "horizontal coarser" response was equal to .5 can therefore be safely rejected. A split-data reliability test was also performed on the grating data by randomly dividing the data of each subject into two groups. These data were then correlated within observers to yield a Pearson (r) coefficient of .7688.

The corresponding data from the line-judgment experiment are presented in Figure 4. This figure 


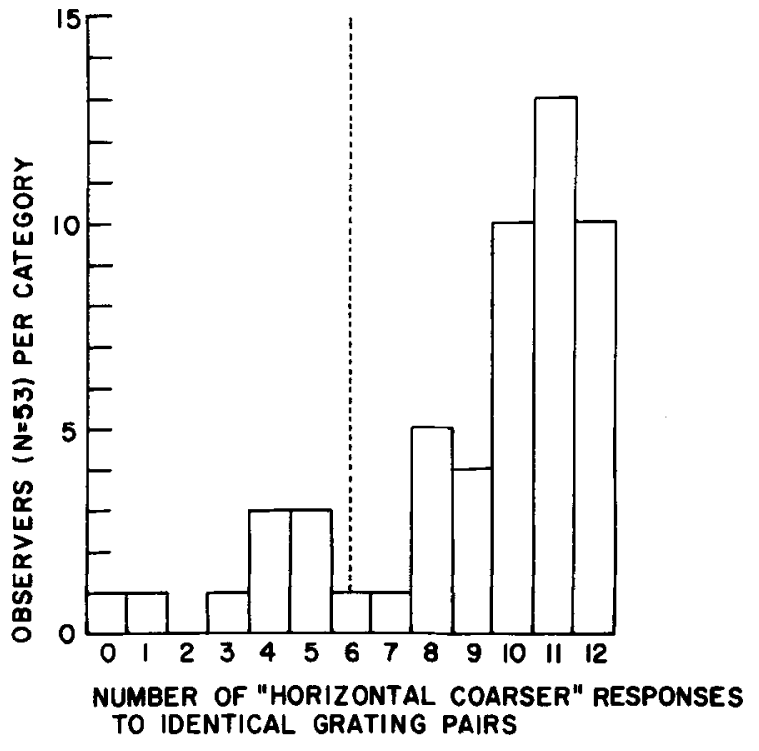

Figure 3. Frequency histogram of number of observers $(n=53)$ choosing horizontal gratings as coarser than vertical gratings a given number of times out of 12 exposures. Spatial frequencies of two gratings were actually the same on these particular exposures. Dashed line represents distribution's expected line of symmetry if choices were governed only by chance.

shows the frequency histogram of observers choosing the horizontal line as longer a given number of times when the two lines were actually the same length. Again, the dashed line indicates the expected center of the response distribution if subjects' responses were governed solely by chance. In this case, the distribution is heavily biased toward the left of this line, signifying that the majority of subjects tested chose the vertical line as longer on a disproportionate number of trials. Again, an expected distribution was computed using a binomial expansion, and this distribution was compared with the one actually obtained (Figure 4). The chi-square statistic equaled 1,125.94 $(\mathrm{df}=9)$. The hypothesis that the probability of a "horizontal longer" response was equal to .5 was rejected with a $\mathrm{p}<.001$. Split data reliability (r) was .4818 .

These orientation-contingent distortions in apparent spatial frequency can also be observed in the error results from the two sets of measurements. Excluding trials in which stimuli were of the same spatial frequency or of the same length, subjects occasionally made errors on those trials in which stimuli were actually different. For instance, if the horizontal line happened to be longer than the vertical line on a particular trial, the observer might mistakenly respond that the vertical line was longer. Total error rates for the grating and line judgments were $4.51 \%$ $(43 / 954)$ and $5.66 \%(48 / 848)$, respectively. Of the 43 errors made during the judgments of gratings, 40 were cases in which observers said the horizontal grating was coarser when, in fact, the vertical grating was coarser. Only three cases of subjects saying the vertical grating was coarser when it was actually finer than the horizontal grating were recorded. A similar bias is found in the line-judgment data. Forty-six of the 48 line-judgment errors were cases in which subjects said the vertical line was longer when the horizontal line was actually longer. Only two errors of the opposite type were recorded.

Therefore, the majority of subjects tested show the presence of the classical horizontal-vertical length illusion. In addition, a significant number of observers tended to see horizontal gratings as coarser than vertical gratings. If these two effects arise from the same influence, they should be correlated within observers. That is, if these effects are due to a compression of the horizontal axis of space relative to the vertical axis, then observers showing a strong bias towards picking vertical lines as the longest should also have a bias towards picking horizontal gratings as coarsest. Thus, the measures shown in Figures 3 and 4 would have an expected correlation of -1.0 . A scatterplot of number of "horizontal-grating-coarser" responses vs. number of "horizontal-line-longer" responses is shown in Figure 5 for the 53 observers. Since both distributions are so heavily skewed, most of the observers fall in the upper left quandrant of Figure 5. Few observers fall in the upper-right and lower-left quadrants, and none fall in the lower-right quadrant. Therefore, no subjects showed both line and grating effects in a direction opposite to that of the majority. Since these distributions are skewed, a Pearson correlation is not entirely appropriate. None-

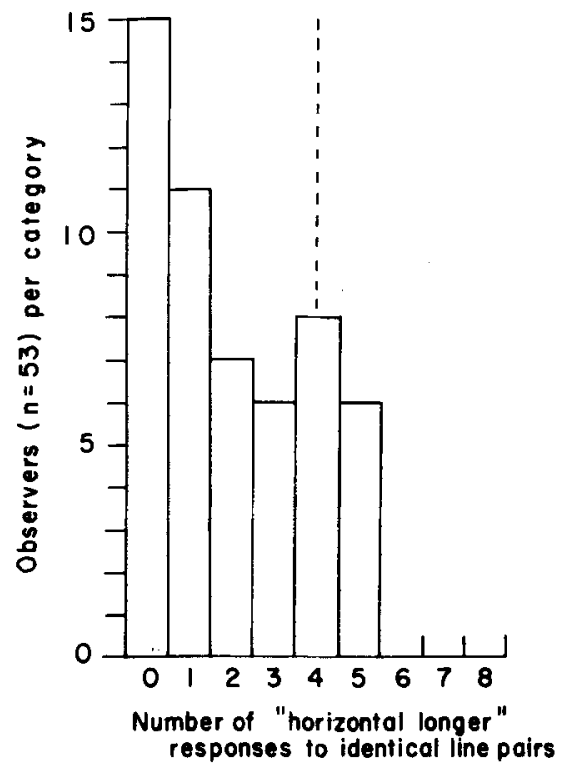

Figure 4. Frequency histogram of number of observers $(n=53)$ choosing horizontal lines as longer than vertical lines a given number of times out of eight exposures. Lines were actually the same length on these trials. Dashed line represents expected line of symmetry if choices were governed by chance alone. 


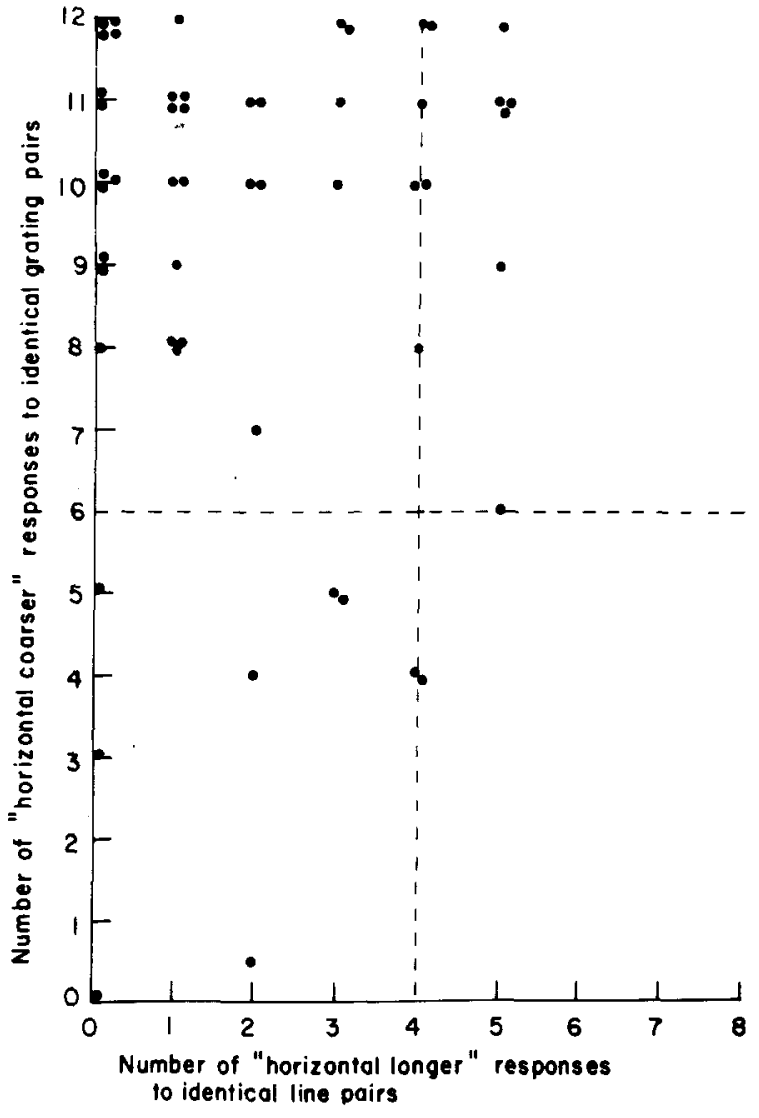

Figure 5. Scatterplot of "horizontal coarser" responses on grating judgment vs. "horizontal longer" responses on line judgments for 53 observers. Dashed lines represent lines of symmetry expected by chance for the distribution. If responses governed only by chance, distribution should be symmetrical about both dashed lines.

theless, such a coefficient was computed and found to be a modest +.0328 . The $99 \%$ confidence intervals for the population correlation coefficient are: -.2444 $\leqslant \mathrm{u} \leqslant+.3100$. A more appropriate measure for testing a relationship between the two sets of data is the Spearman rank-order coefficient. The Spearman coefficient also showed a weak relationship between the two sets of data $(\varrho=+.0650)$. This statistic was converted to a $z$ value of .469 . This value does not allow the rejection of the hypothesis that the population relationship is greater than zero.

\section{DISCUSSION}

Data presented in this paper strongly suggest the presence of orientation-contingent deviations in both apparent length and apparent spatial frequency. For most observers tested, horizontal gratings appear coarser than vertical gratings and horizontal lines appear shorter than vertical lines. Both sets of data are predicted by a model assuming a relative perceptual compression of linear space in the horizontal merid- ian. Therefore, it seems that the two effects may be related to one another. However, when one examines the within-observer correlation of these two sets of judgments, no significant relationship is apparent. Knowing how an observer responded in one part of the experiment does not help in predicting how that observer will respond in the other part of the experiment. Since one cannot prove a null hypothesis, one cannot say that these two measures are uncorrelated in the general population. One can only say that if such a correlation does, in fact, exist, it is in all probability a weak relationship.

It is possible that the weak relationship exhibited between the two sets of data reflects the measurements themselves and does not indicate separate underlying influences for the two phenomena. It is true that most observers reliably show both effects. Perhaps the measures are not sensitive enough to show a relationship. By taking the split-data reliability coefficients, it is possible to correct the sample correlation so that it represents "what the correlation would have been if the two variables had been perfectly measured"' (Guilford \& Fruchter, 1973, pp. 439). When this correction for attenuation is applied, the correlation coefficient rises negligibly from .0328 to .0539. Clearly, this is still much lower than the predicted relationship.

Although no definitive conclusions can be made concerning a common basis for the length and frequency effects, it is clear that the apparent spatial frequency of a suprathreshold grating depends on its orientation. As far as the author is aware, this is the first report of this dependency. The effect of orientation on the perceived spatial frequency of a grating does have consequences for the interpretation of other published data. For instance, Heeley (1979) has reported that spatial frequency adaptation causes shifts in apparent frequency not only about the adaptation orientation but also about orientations orthogonal to the adaptation orientation. This result is in clear contradistinction to earlier reports of Blakemore and Nachmias (1971) and Blakemore, Nachmias, and Sutton (1970). One of the major procedural differences between the two sets of experiments becomes very important in light of the results reported in this paper. While the earlier studies of Blakemore et al. $(1970,1971)$ examined spatial frequency shifts by comparing matches made under conditions of no adaptation to matches made following spatial adaptation, Heeley (1979) collected data only under conditions of adaptation. These data were not compared with baseline matches collected with no adaptation. Thus, when Heeley (1979) had subjects adjust the frequencies of gratings in different orientations to match that of a vertical grating, he was assuming that, with no adaptation, there would be no effect of orientation on perceived spatial frequency. Any match deviations found between the oriented 
test stimuli and the vertical standard were interpreted as arising from the adaptation. The fact that the same spatial frequency does not necessarily appear the same when presented in different orientations poses some problem in the interpretation of data presented by Heeley(1979). Incidentally, a closer examination of Heeley's (1979) data shows that, in all cases, horizontal gratings were adjusted to frequencies higher than the vertical standard, indicating that they actually appeared lower in spatial frequency.

In Bowker (1981), spatial frequency matching data which confirm the orientation-contingent deviations in apparent spatial frequency are reported. In addition, the horizontal-vertical differences are shown to be relatively independent of stimulus spatial frequency and contrast. Finally, these perceptual differences are shown to depend on postoptical factors since they persist when gratings are imaged directly on the retina with laser interference techniques.

\section{REFERENCES}

Appelle, S. Perception and discrimination as a function of stimulus orientation: The "oblique effect" in man and animals. Psychological Bulletin, 1972, 78, 266-278.

Aveny, G. C., \& DAY, R. H. Relationship between the horizontalvertical illusions for velocity and extent. Journal of Experimental Psychology, 1971, 89, 22-31.

Blakemore, C., \& Nachmias, J. The orientation specificity of two visual aftereffects. Journal of Physiology (London), 1971, 213, 157-174.

Blakemore, C., Nachmias, J., \& Sutton, P. The perceived spatial frequency shift: Evidence for frequency-selective neurons in the human brain. Journal of Physiology (London), 1970, 210, 727-750.

BowkER, D. O. Spatial frequency discrimination thresholds in different orientations. Journal of the Optical Society of America, $1980,70,462-463$.

BowkER, D. O. Variations in apparent spatial frequency with stimulus orientation. II. Matching data collected under normal and interferometric viewing conditions. Perception \& Psychophysics, 1981, 29, 568-577.

Brown, J. F. On time perception in visual movement fields. Psychologische Forschung, 1931, 14, 199-232.

Campbelt, F. W., Kulikowski, J. J., \& Levinson, J. The effect of orientation on the visual resolution of gratings. Journal of Physiology (London), 1966, 187, 427-436.
Emsley, H. H. Irregular astigmatism of the eye: Effect of correcting lenses. Transactions of the Optical Society, 1925, 27, $28-41$.

Fick, A. De errore quodam optico asymmetria bulbi effecto. Marburg: Koch, 1851.

Finger, F. W., \& SPELT, D. K. The illustration of the horizontalvertical illusion. Journal of Experimental Psychology, 1947, 37, 243-250.

Freeman, R. D., \& Thibos, L. N. Contrast sensitivity in humans with abnormal visual experience. Journal of Physiology (London), 1975, 247, 687-710.

Guilford, J. P., \& Fruchte R, B. Fundamental statistics in psychology and education (5th ed.). New York: McGraw-Hill, 1973.

HEELEY, D. W. A perceived spatial frequency shift at orientations orthogonal to adapting gratings. Vision Research, 1979, 19, 1229-1236.

Helmholtz, H. Physiological optics (Vol. 3) (trans. J. P. C. Southall). New York: Optical Society of America, (1866, 1910) 1925.

LEIвоWITZ, H. Some observations and theory on the variation of visual acuity with the orientation of the test object. Journal of the Optical Society of America, 1953, 43, 902-905.

Matin, E., \& Drivas, A. Acuity for orientation measured with a sequential recognition task and signal detection methods. Perception \& Psychophysics, 1979, 25, 161-168.

Mitchell, D. E., Freeman, R. D., \& Westheimer, G. Effect of orientation on the modulation sensitivity for interference fringes on the retina. Journal of the Optical Society of America, $1967,57,246-259$.

Ogilvie, J. C., \& TAYloR, M. M. Effect of orientation on the visibility of fine wires. Journal of the Optical Society of America, $1958,48,628-629$.

Pearce, D., \& Matin, L. Variation in the magnitude of the horizontal-vertical illusion with retinal eccentricity. Perception \& Psychophysics, 1969, 6, 241-243.

Pollock, W. T., \& Chapanis, A. The apparent length of a line as a function of its inclination. Quarterly Journal of Experimental Psychology, 1952, 4, 170-178.

RitTer, S. M. The vertical-horizontal illusion. Psychological Monographs, 1917, 23, No. 101.

Shipley, W. C., Nann, B. M., \& Penfield, M. J. The apparent length of tilted lines. Journal of Experimental Psychology, $1949,39,348-351$.

TAYLOR, M. M. Visual discrimination and orientation. Journal of the Optical Society of America, 1963, 53, 763-765.

Thompson, J., \& Schiffman, H. R. The effect on the magnitude of the horizontal-vertical illusion of horizontal retinal eccentricity. Vision Research, 1974, 14, 1463-1465.

WundT, W. Beitrage zur Theorie der Sinneswahrnemungen. Leipzig: Engelmann, 1862.

(Received for publication July 23, 1980; revision accepted January 12, 1981.) 